\title{
Efeitos de Resíduos da Parte Aérea de Sorgo, Milho e Aveia na Emergência e no Desenvolvimento de Plântulas de Leiteiro (Euphorbia heterophylla) RESISTENTES A INIBIDORES DA ALS ${ }^{1}$
}

\author{
Sorghum, Maize and Oat Shoot Residues Affect Emergence and Growth of Euphorbia \\ heterophylla Plants Resistant to ALS-Inhibitors
}

TREZZI, M.M. ${ }^{2}$, VIDAL, R.A. ${ }^{3}$, MATTEI, D. ${ }^{4}$, SILVA, H.L. ${ }^{4}$, CARNIELETO, C.E. ${ }^{4}$, GUSTMANN,
M.S. ${ }^{4}$, VIOLA, R. ${ }^{4}$ e MACHADO, A. ${ }^{4}$

\begin{abstract}
RESUMO - Populações de Euphorbia heterophylla resistentes (R) aos herbicidas inibidores da enzima acetolactato sintase encontram-se amplamente disseminadas em várias regiões do Brasil. Resíduos culturais de várias espécies apresentam elevado potencial para suprimir o desenvolvimento de plantas daninhas. O objetivo deste trabalho foi avaliar os efeitos de diferentes níveis de resíduos das partes aéreas de sorgo, milho e aveia na supressão de E. heterophylla resistente a herbicidas. Foram realizados dois experimentos em vasos com delineamento experimental completamente casualizado, com quatro repetições. No primeiro, os tratamentos foram arranjados em fatorial $4 \times 6$, em que o fator A representou três híbridos de sorgo (AG-2501, Dow 1P-400 e BR-501) e um de milho (AG-3010); e o fator B, seis níveis de resíduos na superfície do solo $\left(0,3,25,6,5,9,75,13\right.$ e $\left.26 \mathrm{t} \mathrm{ha}^{-1}\right)$. Os tratamentos do segundo experimento foram arranjados em fatorial $2 \times 6$, em que A representou as espécies (sorgo e aveia) e B os níveis de resíduos $(0,1,12,3,25,6,5,13$ e 26 t ha-1 $)$. A emergência das plantas de E. heterophylla foi atrasada e reduzida. Estimou-se que seriam necessárias $28,6 \mathrm{t} \mathrm{ha}^{-1}$ de palha de sorgo para causar redução de $50 \%$ na emergência de plântulas. O número de folhas diminuiu com a elevação dos níveis de resíduos da parte aérea de sorgo e milho. Niveis intermediários de resíduos de Dow 1P-400 reduziram mais o IVE do que os outros híbridos de sorgo, entretanto não houve diferenças entre genótipos quanto à emergência de plântulas. Resíduos da parte aérea de aveia não foram eficazes em reduzir a emergência de $E$. heterophylla.
\end{abstract}

Palavras-chave: resistência, níveis de palha, velocidade de emergência, milho e sorgo.

ABSTRACT - Euphorbia heterophylla populations resistant to ALS-inhibitors were reported in many regions in Brazil. Straw residues of many species have shown high potential to supress weeds. The objective of this work was to evaluate the efficacy of different rates of sorghum, corn and black oat shoot residues in supressing emergence and growth of $\boldsymbol{E}$. heterophylla resistant to herbicides. Two experiments were conducted in pots in a completely randomized design, with four replicates, arranged in a factorial scheme. In the first trial, factor A was composed by sorghum (AG-2501, Dow 1P-400 and Br-501) and corn (AG-3010) hybrids and factor B by six rates of sorghum and corn residues above soil surface $\left(0,3.25,6.5,9.75,13\right.$ and $\left.26 \mathrm{th} \mathrm{h}^{-1}\right)$. In the second trial, factor A was composed by two species (sorghum and oat) and factor B by shoot residue rates $\left(0,1.12,3.25,6.5,13\right.$ and $\left.26 t \mathrm{ha}^{-1}\right)$. Emergence of $\boldsymbol{E}$. heterophylla plants was delayed and decreased. The total number of leaves decreased with increase of sorghum and corn straw rate. However, it was estimated that $28.6 \mathrm{tha}^{-1}$ of sorghum residues would be necessary to decrease $50 \%$ emergence. Dow $1 P-400$ sorghum straw at intermediate rates presented a greater potential to decrease IVE than the other sorghum hybrids, but no difference was observed between emergence of sorghum genotypes. Black oat shoot straw was not efficient in decreasing $\mathbf{E}$. heterophylla emergence.

Keywords: resistance, straw rates, emergence velocity, corn and sorghum.

Recebido para publicação em 7.2.2006 e na forma revisada em 4.8.2006.

Eng.-Agrônomo, Dr., Curso de Agronomia - UTFPR, Campus Pato Branco,Via do Conhecimento, km 01, Caixa postal 571, 85503-390 Pato Branco-PR. ${ }^{2}$ Eng.-Agrônomo, Ph.D., Faculdade de Agronomia - UFRGS, Av. Bento Gonçalves, 7712, Caixa Postal 776, 15100-000 Porto Alegre-RS. ${ }^{3}$ Acadêmico do Curso de Agronomia - UTFPR, Campus Pato Branco.

Planta Daninha, Viçosa-MG, v. 24, n. 3, p. 443-450, 2006 


\section{INTRODUÇÃO}

O leiteiro ou amendoim-bravo (Euphorbia heterophylla) é uma planta daninha da família Euphorbiaceae, originária da América Tropical e Subtropical, amplamente disseminada em todo o Brasil (Kissmann \& Groth, 1992), causando muitos danos na região sudoeste do Paraná.

A grande capacidade de produção de sementes e a emergência em fluxos são fatores que dificultam o manejo dessa espécie, pois propiciam o reabastecimento do banco de sementes do solo, perpetuando a infestação das áreas. Dez plantas de E. heterophylla em um $\mathrm{m}^{2}$ são capazes de reduzir em $7 \%$ o rendimento de grãos quando o período de convivência com a cultura ocorre durante todo o ciclo (Chemale \& Fleck, 1982). Qualquer mecanismo capaz de reduzir a germinação das sementes do banco de sementes pode constituir-se em estratégia útil no controle dessa espécie (Suda \& Pereira, 1997).

Hoje, no Brasil, estão catalogadas 14 espécies de plantas daninhas resistentes (R) aos herbicidas, pertencentes a cinco mecanismos de ação (inibidores da ALS, inibidores da ACCase, auxinas sintéticas, inibidores da EPSPS e inibidores da PROTOX), sendo oito delas resistentes a herbicidas inibidores da ALS (Heap, 2005). Entre essas oito, E. heterophylla pode ser considerada a mais difundida e a que causa maiores prejuízos.

Em áreas de soja com E. heterophylla resistente a inibidores da ALS, poucos são os herbicidas seletivos alternativos a esse mecanismo de ação que exercem controle eficiente sobre essa espécie. A utilização de herbicidas inibidores da PROTOX, principal mecanismo alternativo para o controle de E. heterophylla em soja, implica muitas vezes os chamados "escapes", pois torna-se muito difícil controlar todas as plantas de E. heterophylla no estádio recomendado (duas folhas verdadeiras), mesmo fazendo-se mais de uma aplicação herbicida em pós-emergência. A utilização de herbicidas desse mecanismo de ação após o estádio recomendado interrompe a dominância apical e favorece a brotação das gemas laterais de plantas de E. heterophylla.

Coberturas mortas de espécies como sorgo e aveia apresentam capacidade supressora da emergência de plantas daninhas. Quantidades de palha de sorgo de 1,5 e 1,1 $\mathrm{tha}^{-1}$ foram suficientes para reduzir as infestações de Sida rhombifolia (SIDRH) e Brachiaria plantaginea (BRAPL) em 50\%, comparativamente à testemunha sem cobertura morta na superfície. Além disso, palha de sorgo na quantidade de $4 \mathrm{t} \mathrm{ha}^{-1}$ na superfície do solo reduziu a densidade de SIDRH em 93\% e a de BRAPL em 96\% (Trezzi \& Vidal, 2004). A cobertura morta de aveia-preta foi capaz de diminuir a densidade de plantas de BRAPL, aumentando o rendimento da soja (Theisen et al., 2000). A presença de aveia-preta reduziu a taxa de emergência das sementes de E. heterophylla presentes no banco, porém permitiu que as plantas se estabelecessem (Seifert \& Voll, 2000). Já a presença de cobertura com palha de cana-deaçúcar em níveis crescentes de 2 a $15 \mathrm{t} \mathrm{ha}^{-1}$ não foi suficiente para reduzir a emergência de E. heterophylla (Martins et al., 1999). O milho também apresenta potencial de redução de emergência de plantas daninhas, principalmente gramíneas; para cada tonelada de palha houve redução de 6,4 e 4,2\% da emergência de plantas daninhas, respectivamente, aos 26 e 41 dias após a semeadura de milho (Oliveira et al., 2001)

Os efeitos da palhada sobre as plantas daninhas provêm de efeitos físicos ou alelopáticos, sendo difícil diferenciar um do outro em campo, já que ambos ocorrem de forma simultânea (Maciel et al., 2003). Os efeitos físicos da palha se devem principalmente ao sombreamento do solo, à barreira física para a emergência da plântula da espécie daninha e à manutenção de temperaturas do solo mais baixas, em relação ao solo descoberto (Aase et al., 1991; Teasdale \& Mohler, 1993; Vidal, 1995).

Os mecanismos envolvendo a germinação de sementes de $E$. heterophylla ainda não foram completamente esclarecidos, havendo necessidade de mais informações na literatura científica. Alternâncias de temperatura de $25 / 30{ }^{\circ} \mathrm{C}$, em laboratório, estimulam a germinação de E. heterophylla (Bannon et al., 1978). Em geral, considera-se que E. heterophylla apresenta fotoblastismo positivo, não germinando sob ausência de radiação (Suda \& Pereira, 1997). No entanto, as condições ambientais de temperatura e fotoperíodo durante 
o desenvolvimento da planta-mãe e a idade da planta genitora influenciam essa característica (Suda \& Pereira, 1997).

É importante frisar que existe variabilidade genética quanto à produção de compostos alelopáticos entre genótipos de plantas cultivadas (Ben Hammonda et al., 1995; Jacobi, 1997; Trezzi et al., 2005) e que biótipos resistentes de E. heterophylla também apresentam variabilidade genética elevada (Winkler et al., 2003), podendo exibir variação na suscetibilidade aos efeitos da presença da palhada na superfície do solo.

Este trabalho teve por objetivo avaliar os efeitos de diferentes níveis de resíduos das partes aéreas de genótipos de sorgo, milho e aveia sobre a supressão de plantas de um biótipo de E. heterophylla resistente a herbicidas inibidores de ALS.

\section{MATERIAL E MÉTODOS}

Para verificação do efeito do uso de coberturas mortas sobre a emergência e o desenvolvimento de plantas de E. heterophylla, foram conduzidos dois experimentos em casa de vegetação pertencente ao Curso de Agronomia da UTFPR, localizada no município de Pato Branco-PR, nos anos de 2004 e 2005. O primeiro experimento comparou diferentes genótipos de sorgo e de milho, e o segundo, os efeitos de sorgo e aveia.

Plantas de sorgo, milho e aveia foram coletadas a campo, no estádio de florescimento, e levadas para casa de vegetação, onde, após período de pré-murchamento, passaram por triturador de lâminas e martelos. Posteriormente, o material foi seco ao sol e revolvido, para uniformizar a secagem.

Sementes de E. heterophylla com resistência aos herbicidas inibidores da ALS foram coletadas em propriedade localizada no município de Mamborê (PR). Foram utilizados vasos com capacidade para $8 \mathrm{~kg}$, e o substrato utilizado foi o solo Latossolo Vermelho Distrófico, coletado em área sem histórico de infestação de E. heterophylla. Aleatoriamente, distribuíram-se 50 sementes de E. heterophylla em cada vaso, a aproximadamente $0,5 \mathrm{~cm}$ de profundidade. As quantidades de resíduos vegetais foram pesadas, corrigidas tendo como referência a base seca e, posteriormente, distribuídas na superfície do solo.

O delineamento experimental utilizado nos dois experimentos foi o completamente casualizado, com quatro repetições. Os tratamentos do primeiro experimento foram arranjados em fatorial $4 \times 6$, em que o fator $A$ incluiu três genótipos de sorgo (AG-2501, BR-501 e Dow 1P-400) e o híbrido de milho Ag 3010. O fator $\mathrm{B}$ correspondeu aos niveis de matéria seca de resíduos da parte aérea desses materiais: $0,3,25,6,5,9,75,13$ e $26 \mathrm{t} \mathrm{ha}^{-1}$. O segundo experimento foi arranjado em um fatorial 2x6, sendo o fator A a espécie vegetal, constituída por sorgo, híbrido Dow 1P-400 e aveia-preta comum, e o fator $\mathrm{B}$, os niveis de palha $\left(0,1,12,3,25,6,5,13\right.$ e $\left.26 \mathrm{t} \mathrm{ha}^{-1}\right)$.

Nos dois experimentos, foi realizada a contagem diária de plântulas de $E$. heterophylla emergidas na superfície da camada de palha, até que cessasse a sua emergência. A palha não foi movimentada para a contagem, pois incidência de luz decorrente da movimentação da palha poderia afetar a emergência de plântulas. Esse procedimento permitiu a determinação do percentual total de plantas emergidas por área e o cálculo do índice de velocidade de emergência (IVE), pelo somatório dos quocientes determinados pela diferença entre o número de plântulas emergidas em avaliações sucessivas e o número total de dias desde o início da emergência delas.

Apenas no primeiro experimento, aos oito dias após o início da emergência (DIE) de $E$. heterophylla, foram contadas as folhas de dez plântulas, aleatoriamente em cada vaso. Para isso, os cotilédones foram considerados como folhas, pares de folhas não completamente expandidas como uma e pares de folhas completamente expandidas foram contados como duas folhas. Aos 16 DIE, a mesma avaliação foi feita em todas as plantas de cada vaso.

Aos 16 DIE, no primeiro experimento, a parte aérea de E. heterophylla foi seca em estufa a $60{ }^{\circ} \mathrm{C}$, até atingir peso constante, para determinação da matéria seca.

Realizou-se a análise da variância dos dados pelo teste $\mathrm{F}$ e, em caso de significância, os dados de genótipo/espécie foram submetidos à comparação de médias pelo teste de 
Tukey a 5\% de probabilidade; os dados de níveis de palha foram analisados por regressão polinomial.

\section{RESULTADOS E DISCUSSÃO}

No experimento em que se compararam resíduos de sorgo e milho, a análise da variância indicou interação entre os fatores genótipo e níveis de palha, no tocante à velocidade de emergência (IVE). Em todos os genótipos de sorgo e milho, o IVE foi reduzido linearmente em função do aumento dos resíduos na superfície, com exceção do genótipo de sorgo Dow 1P-400, o qual apresentou melhor ajuste pelo modelo quadrático. No nível de palha de 9,75 t ha ${ }^{-1}$, os resíduos do sorgo Dow 1P-400 atrasaram a emergência de E. heterophylla de forma mais eficaz do que os demais genótipos de sorgo e milho (Figura la e Tabela 1). No nível de 6,75 t ha-1, o efeito da palha de Dow 1P-400 foi superior apenas ao do milho (Figura la e Tabela 1).

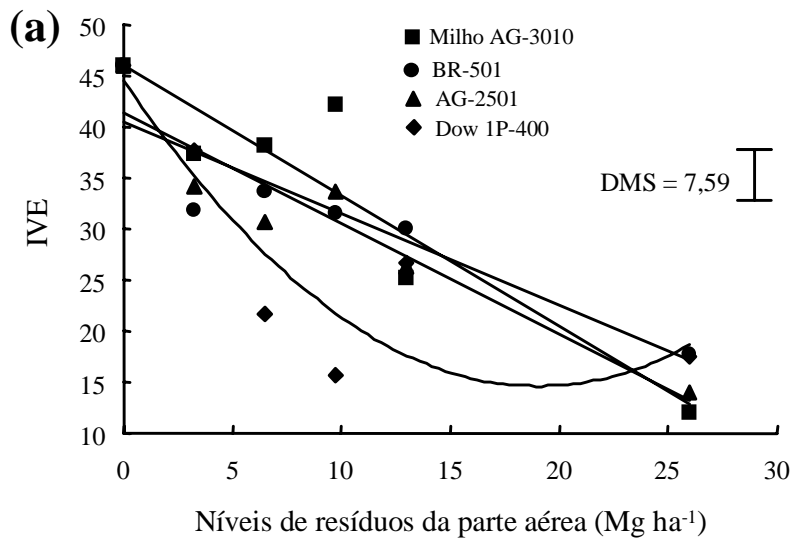

Quanto ao número de plantas emergidas, ocorreram apenas efeitos dos níveis de palha, não havendo efeitos de genótipo nem interação entre os fatores. A redução na emergência das plantas foi linear com a elevação dos niveis de palha na superfície (Figura 1b). A partir da equação linear, estimou-se que haveria necessidade de 28,6 t ha ${ }^{-1}$ de palha de sorgo ou milho para inibir em 50\% a emergência das plântulas de E. heterophylla. Esses valores são muito superiores aos niveis de resíduos de sorgo (apenas 1,5 e 1,1 t ha-1, respectivamente) necessários para decrescer, em 50\%, as infestações de Sida rhombifolia e de Brachiaria plantaginea (Trezzi \& Vidal, 2004).

No experimento comparando aveia-preta comum com sorgo Dow 1P-400, para a variável emergência de plantas, houve interação entre niveis de palha e espécie. Com o incremento dos níveis de palha de sorgo na superfície do solo, houve redução linear da emergência de plântulas de E. heterophylla. Contudo, a emergência desta espécie não foi afetada pelo

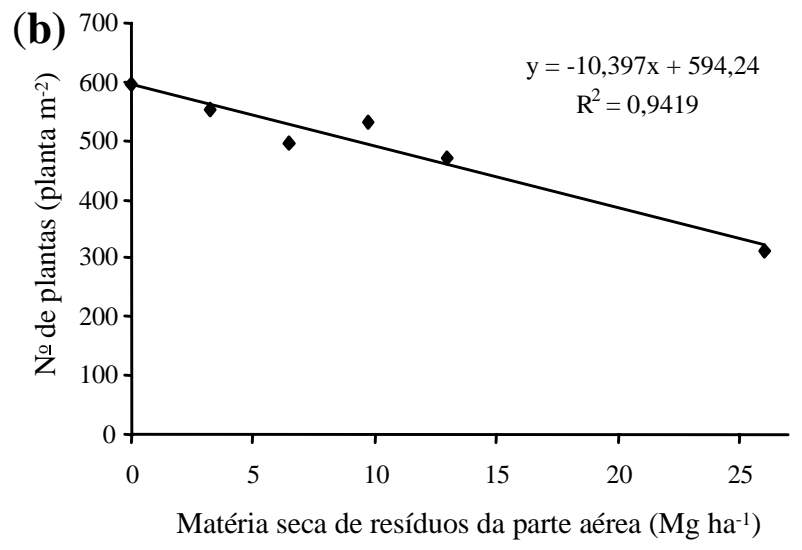

Figura 1 - Efeitos de níveis de palha sobre: (a) IVE (índice de velocidade de emergência); e (b) emergência total de plântulas de E. heterophylla (média dos genótipos de sorgo e de milho).

Tabela 1 - Equações e coeficientes de determinação das regressões linear e quadrática entre níveis de palha e IVE de plântulas de Euphorbia heterophylla

\begin{tabular}{|c|c|c|c|}
\hline Genótipo & Regressão Linear & Regressão Quadrática & $\mathrm{R}^{2}$ \\
\hline BR-501 & $y=-1,28 x+45,9$ & --- & 0,86 \\
\hline AG-2501 & $y=-1,085 x+41,417$ & --- & 0,89 \\
\hline Dow-1P-400 & 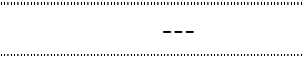 & $y=0,0828 x^{2}-3,1516 x+44,546$ & 0,77 \\
\hline Milho & $y=-1,2758 x+45,924$ & --- & 0,86 \\
\hline
\end{tabular}


incremento dos níveis de aveia-preta (Figura 2a). Trabalhando com cana-de-açúcar, Martins et al. (1999) verificaram resultados similares aos obtidos com resíduos de aveia, pois o uso de resíduos na faixa de 2 a $15 \mathrm{t} \mathrm{ha}^{-1}$ não reduziu a emergência dessa espécie.

Em relação ao IVE, apenas houve efeito significativo de níveis de palha, não havendo diferenças significativas entre os resíduos de sorgo Dow 1P-400 e aveia-preta comum, pois ambos foram capazes de reduzir a velocidade de emergência com o aumento dos níveis de palha na superfície do solo (Figura 2b). Maciel et al. (2003) não observaram efeitos de níveis de Brachiaria decumbens sobre a velocidade de emergência de $E$. heterophylla. No entanto, eles utilizaram no máximo $6 \mathrm{t}$ ha ${ }^{-1}$ de resíduos da parte aérea dessa espécie na superfície do solo.

A redução do IVE e do número total de plantas emergidas com o aumento dos níveis de palha pode ser explicada pelo sombreamento do solo e pela manutenção de menor amplitude térmica nessas condições (dados não avaliados). A ausência de luz pode inibir a germinação de E. heterophylla (Suda \& Pereira, 1997).

As diferenças entre os genótipos de sorgo e milho, nos niveis de 6,5 a 9,75 tha-1 de palha (Figura 1a), em geral, são atribuídas a efeitos alelopáticos diferenciais das espécies cultivadas ou genótipos sobre as plantas daninhas (Ben Hammonda et al., 1995; Jacobi, 1997; Trezzi et al., 2005). Elas sugerem maior concentração de compostos alelopáticos nos resíduos do genótipo de sorgo Dow 1P-400, ou mesmo a presença de compostos mais ativos nesse genótipo, quando comparado aos demais. Esses resultados concordam com os obtidos por Trezzi et al. (2005), que verificaram diferenças entre genótipos de sorgo quanto à capacidade de produzir efeito alelopático de extratos de natureza hidrofílica, provenientes da parte aérea e dos sistemas radiculares das plantas. Correia et al. (2005) relataram que extratos foliares de alguns híbridos de sorgo foram capazes de inibir o crescimento radicular da soja em até $54 \%$ e demonstraram ausência de efeito inibitório de extratos de raízes e caule.

Com o incremento dos níveis de resíduos de todos os genótipos de sorgo na superfície do solo, observou-se redução no número de folhas de E. heterophylla, quando avaliado aos oito dias do início da emergência (DIE) (Figura 3a e Tabela 2). Aos 16 DIE, apenas os resíduos de Dow 1P-400 e AG-2501 reduziram o número de folhas de E. heterophylla, em comparação à testemunha sem resíduos na superfície (Figura 3b e Tabela 3). Maciel et al. (2003) não observaram efeitos de $6 \mathrm{t} \mathrm{ha}^{-1}$ de resíduos da parte aérea de $B$. decumbens sobre a área foliar de plantas de E. heterophylla.
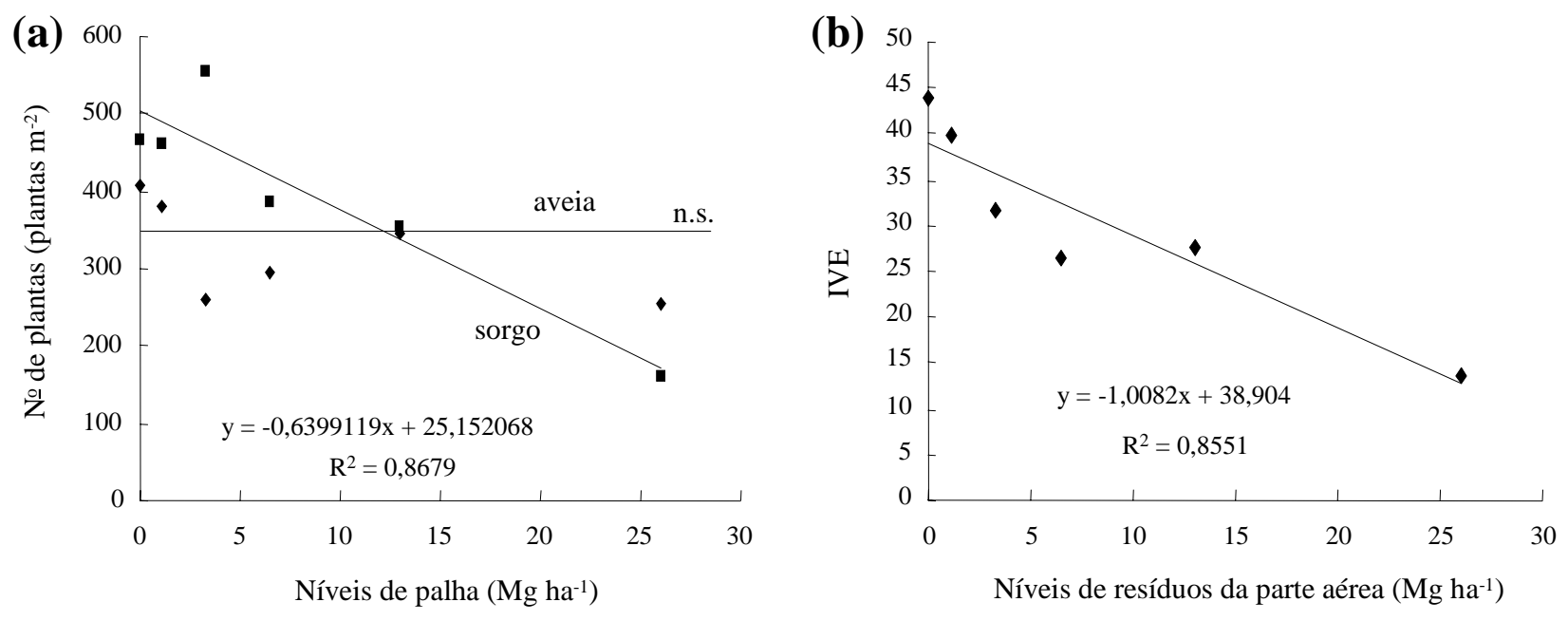

Figura 2 - Efeito de resíduos da parte aérea de sorgo e de aveia sobre: (a) emergência de plântulas de E. heterophylla; e (b) índice de velocidade de emergência (IVE) de E. heterophylla (dados médios das duas espécies). 


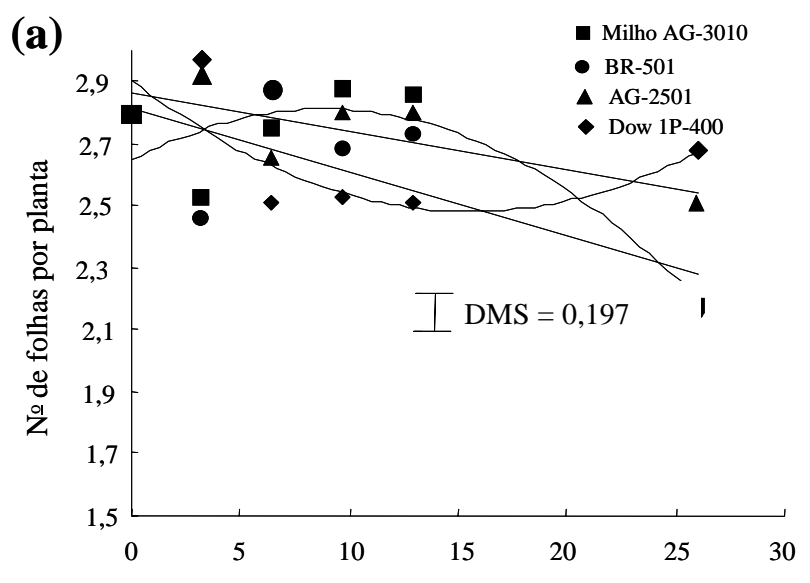

Níveis de resíduos da parte aérea $\left(\mathrm{Mg} \mathrm{ha}^{-1}\right)$

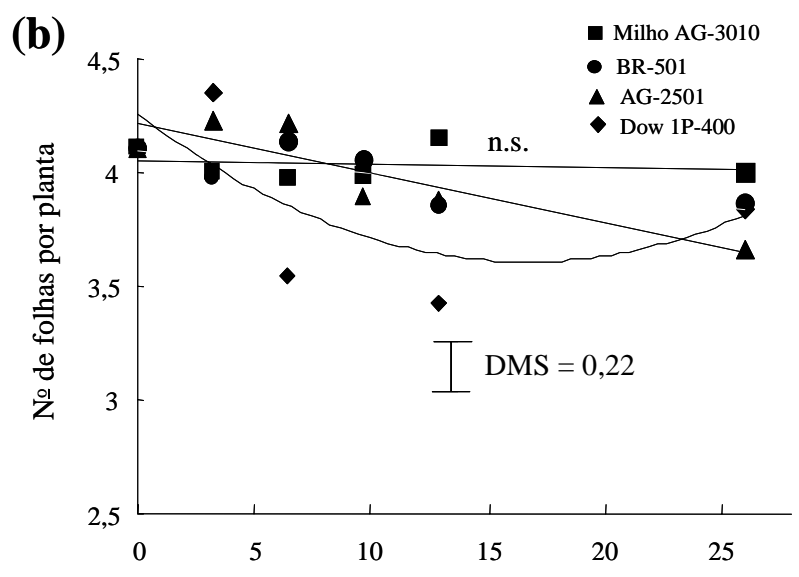

Níveis de resíduos da parte aérea $\left(\mathrm{Mg} \mathrm{ha}^{-1}\right)$

Figura 3 - Efeitos de níveis de palha e de genótipos de sorgo sobre o número de folhas de E. heterophylla aos 8 (a) e aos 16 dias após o início da emergência (b).

Tabela 2 - Equações e coeficientes de determinações das regressões linear e quadrática entre níveis de palha e número de folhas de plântulas de Euphorbia heterophylla aos 8 DIE

\begin{tabular}{|c|c|c|c|}
\hline Genótipo & Regressão Linear & Regressão Quadrática & $\mathrm{R}^{2}$ \\
\hline BR-501 & $y=-0,0208 x+2,815$ & --- & 0,5 \\
\hline AG-2501 & $y=-0,0123 x+2,8$ & --- & 0,57 \\
\hline Dow-1P-400 & 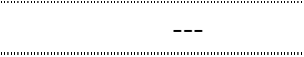 & $y=0,0017 x^{2}-0,054 x+2,902$ & 0,58 \\
\hline Milho & 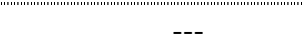 & $y=-0,0021 x^{2}+0,0373 x+2,6465$ & 0,77 \\
\hline
\end{tabular}

Tabela 3 - Equações e coeficientes de determinações da regressão linear entre níveis de palha e número de folhas de plântulas de Euphorbia heterophylla aos 16 DIE

\begin{tabular}{|c|c|c|c|}
\hline Genótipo & Regressão Linear & Regressão Quadrática & $\mathrm{R}^{2}$ \\
\hline BR-501 & --- & --- & --- \\
\hline AG-2501 & $y=-0,0216 x+4,2101$ & --- & 0,78 \\
\hline Dow-1P-400 & --- & $y=0,0023 x^{2}-0,0777 x+4,2566$ & 0,4 \\
\hline Milho & 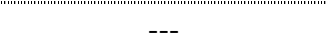 & --- & "'-"- \\
\hline
\end{tabular}

Na prática, a diminuição da velocidade de emergência associada à redução do número de folhas poderia aumentar o período de tempo para a realização do controle químico de E. heterophylla, o que tornaria mais eficiente essa operação, principalmente em áreas com problemas de resistência a herbicidas inibidores da ALS. Os herbicidas inibidores da
PROTOX são considerados as principais alternativas químicas de controle de E. heterophylla, pois controlam eficientemente esta espécie com no máximo duas folhas verdadeiras (Vidal \& Merotto Jr., 2001) e têm um período ideal de aplicação relativamente curto, que poderia ser aumentado pela presença de palha de sorgo. 


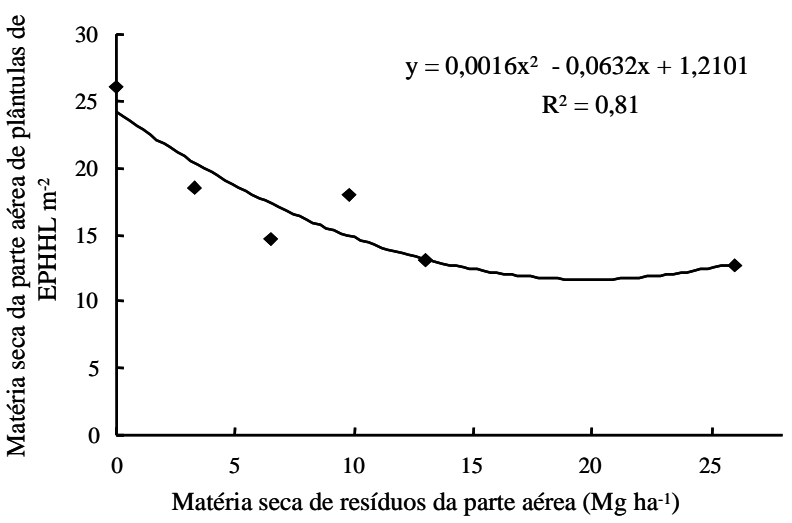

Figura 4 - Efeitos de níveis de resíduos da parte aérea (média de sorgo e milho) sobre a matéria seca de plântulas de E. heterophylla.

A matéria seca total da parte aérea de plântulas de E. heterophylla sofreu efeitos apenas dos níveis de palha de sorgo e milho, não ocorrendo diferenças entre genótipos / espécie (Figura 4). Como não houve efeito sobre a matéria seca de cada planta (dados não mostrados), conclui-se que o número de folhas de E. heterophylla é um indicador mais adequado para se avaliarem os efeitos negativos dos resíduos de sorgo e milho do que a matéria seca das plantas.

O presente trabalho mostrou que a presença de palha de sorgo, milho ou aveia na superfície do solo pode reduzir a velocidade de emergência de $E$. heterophylla, o que poderia beneficiar a intervenção com métodos químicos. Entretanto, apenas os resíduos de milho e sorgo foram capazes de reduzir a emergência total dessa espécie daninha. Niveis de palha de sorgo e milho necessários para suprimir totalmente a emergência de $E$. heterophylla são muito elevados e difíceis de serem obtidos em lavouras destinadas à comercialização de grãos. Foram constatadas diferenças de capacidade de supressão de E. heterophylla em função da espécie de cobertura morta ou do genótipo utilizado, e o sorgo Dow-1P-400 demonstrou o maior potencial entre os genótipos avaliados.

\section{LITERATURA CITADA}

AASE, J. K.; TANAKA, D. L. Reflectances from four wheat residue cover densities as influenced by three soil backgrounds. Agron. J., v. 83, n. 3, p. 753-757, 1991.
BANNON, J. S.; BARKER, J. B.; ROGERS, R. L. Germination of wild poinsettia (Euphorbia heterophylla). Weed Sci., v. 26, n. 3, p. 221-225, 1978.

BEN-HAMMOUDA, M. et al. A chemical basis for differential allelopathic potencial of sorghum hybrids on wheat. J. Chem. Ecol., v. 21, n. 6, p. 775-786, 1995.

CHEMALE, V. M.; FLECK, N. G. Avaliação de cultivares de soja (Glycine max L. Merril) em competição com Euphorbia heterophylla $\mathrm{L}$. sob três densidades e dois períodos de ocorrência. Planta Daninha, v. 5, p. 36-45, 1982.

CORREIA, N. M.; CENTURION, M. A.; ALVES, P. L. Influência de extratos aquosos de sorgo sobre a germinação e desenvolvimento de plântulas de soja. Ci. Rural, v. 35, p. 498-503, 2005.

HEAP, I. International survey of herbicide-resistant weeds. Disponível em: 〈www.weedscience.org >. Acesso em: 12 nov. 2005.

JACOBI, U. S. Avaliação do potencial alelopático de genótipos de aveia. 1997. $165 \mathrm{f}$. Tese (Doutorado em Fitotecnia) - Universidade Federal do Rio Grande do Sul, Porto Alegre, 1997.

KISSMANN, K. G.; GROTH, D. Plantas infestantes e nocivas. São Paulo: Basf, 1992, 798 p. t. 2.

MACIEL, C. D. G. et al. Influência do manejo da palhada de capim-braquiária (Brachiaria decumbens) sobre o desenvolvimento inicial de soja (Glycine Max) e amendoimbravo (Euphorbia heterophylla). Planta Daninha, v. 21, n. 3, p. 365-373, 2003.

MARTINS, D. et al. Emergência em campo de dicotiledôneas infestantes em solo coberto com palha de cana-de-açúcar. Planta Daniha, v. 17, p. 151-161, 1999.

OLIVEIRA, M. F. et al. Efeito da palha e da mistura atrazine e metolachlor no controle de plantas daninhas na cultura do milho, em sistema de plantio direto. Pesq. Agropec. Bras., v. 36, n. 1 , p. 37-41, 2001.

SEIFERT, G.; VOLL, E. Cobertura de aveia e calagem sobre amendoim-bravo em semeadura direta de soja. Planta Daninha, v. 18, n. 2, p. 309-322, 2000.

SUDA, C. N. K.; PEREIRA, M. F. D. A. Sensibilidade à luz de sementes de Euphorbia heterophylla L. durante a germinação. R. Bras. Fisiol. Vegetal, v. 9, n. 1, p. 55-64, 1997.

TEASDALE, J. R.; MOHLER, C. L. Light transmittance, soil temperature, and soil moisture under residue of hairy vetch and rye. Agron. J., v. 85, n. 3, p. 673-680, 1993. 
THEISEN, G.; VIDAL, R. A.; FLECK, N. G. Redução da infestação de Brachiaria plantaginea em soja pela cobertura do solo com palha de aveia-preta. Pesq. Agropec. Bras., v. 35, n. 4, p. 753-756, 2000.

TREZZI, M. M.; VIDAL, R. A. Potencial de utilização de cobertura vegetal de sorgo e milheto na supressão de plantas daninhas em condição de campo: II - Efeitos da cobertura morta. Planta Daninha, v. 22, n. 1, p. 1-10, 2004.

TREZZI, M. M.; VIDAL, R. A.; KRUSE, N. D.

Fitotoxicidade de extratos hidrofóbicos e hidrofílicos de sorgo e milheto. R. Bras. Ci. Agrovet., v. 4, n. 1, p. 25-34, 2005.
VIDAL, R. A. Amount of crop residues in no-till farming affects weed-crop ecosystems. 1995. $161 \mathrm{f}$. Thesis (Ph.D.) - Purdue University, West Laffayete, 1995.

VIDAL, R. A.; MEROTTO JR., A. Herbicidas inibidores da PROTOX. In: VIDAL, R. A.; MEROTTO JR., A. (Ed.). Herbicidologia. Porto Alegre: UFRGS, 2001. p. 69-86.

WINKLER, L. M.; VIDAL, R. A.; NETO, J. F. B. Caracterização genética de Euphorbia heterophylla resistente aos herbicidas inibidores da acetolactato sintase. Pesq. Agropec. Bras., v. 38, n. 9, p. 1067-1072, 2003. 